\title{
Activated bentonite promoted Friedländer condensation reactions: Synthesis of thieno[2,3-b]quinolinones and tacrines analogues derivatives
}

\author{
Khaireddine Mohamed Dridi a,b,*, Ridha Ben Said a,c, \\ Youssef Arfaoui a,c and Abdullah Sulaiman Al-Ayed a \\ a Department of Chemistry, College of Sciences and Arts, Qassim University, Al-Rass, 53, Kingdom of Saudi Arabia \\ b Laboratory of Organic Chemistry, Department of Chemistry, Faculty of Sciences, El Manar University, El Manar II, Tunis 1060, Tunisia \\ c Unité Physico-Chimie des Matériaux Condensés, Département de Chimie, Faculté des Sciences, Université Tunis El Manar, El Manar II, Tunis 1060, Tunisia \\ ${ }^{*}$ Corresponding author at: Department of Chemistry, College of Sciences and Arts, Qassim University, Al-Rass, 53, Kingdom of Saudi Arabia. \\ Tel.: +966.05.59928442; fax: +966.06.3335160. E-mail address: khairydridi@yahoo.fr (K.M. Dridi).
}

\section{ARTICLE INFORMATION}

Received: 01 April 2013

Received in revised form: 25 April 2013

Accepted: 08 May 2013

Online: 30 September 2013

\section{KEYWORDS}

\section{Ketones}

Bentonite

Tacrine analogues

Friedlander reaction

2-Aminothiophene-3-carbonitrile

4-Aminothieno[2,3-b]quinolinones

\section{Introduction}

Tacrine (Figure 1) sold under the name Gognex has been the first drug that proved to have a beneficial effect on cognition in patients with Alzheimer's disease (AD) [1,2]. However this drug is not used anymore due to its side effects such as hepatotoxicity [3,4]. In order to investigate the biological effects of structurally modified tacrine and as an extension of our efforts towards the facile synthesis of heterocycles containing thiophene ring moiety $[5,6]$. We report here the synthesis of series of thiophene analogues, since it is widely recognized that thiophene ring is a bioisostere of benzene. 2-Aminothiophene-3-carbonitrile, 1, which were found to possess an extensive spectrum of pharmacological activities [7,8-10], were chosen as synthon for the synthesis of 4-aminothienoquinolinones $\mathbf{2}$ and thienotacrine, $\mathbf{3}$.

\section{Experimental}

\subsection{Instrumentation}

Melting points were taken with a Kofler hot staged apparatus and are uncorrected. All reactions were monitored by thin layer chromatography (TLC). IR spectra were determined with a Perkin Elmer 1600 series FT-IR spectrometer. ${ }^{1} \mathrm{H}$ and ${ }^{13} \mathrm{C}$ NMR spectra were recorded on a Varian-Unity spectrometer at $300 \mathrm{MHz}$ using TMS as an internal standard. Elemental analyses were determined using a Elementar Vario EI III Elemental Analyser

\subsection{Synthesis}

\subsubsection{General procedure for the synthesis of starting material substituted 2-aminothiophene-3-carbonitrile (1)}

In a typical experiment, ketone $(0.10 \mathrm{~mol})$ and malononitrile $(0.10 \mathrm{~mol})$ were dissolved in $200 \mathrm{~mL}$ of absolute ethanol. Sulphur powder $(0.11 \mathrm{~mol})$ and morpholine $(20 \mathrm{~mL})$ were added. The mixture was heated at $50^{\circ} \mathrm{C}$ during 3 hours and then was cooled at room temperature. The mixture poured into $300 \mathrm{~mL}$ ice-water. The filtered precipitate was washed with cold water, dried and then recrystallized in suitable solvent (Scheme 1).

2-Amino-4,5,6,7-tetrahydrobenzo[b]thiophene-3-carbonitrile (1a): Color: Ocher needles. Yield: 92 \%. M.p.: $145-147^{\circ} \mathrm{C}$. FT-IR $\left(\mathrm{CHCl}_{3}, v, \mathrm{~cm}^{-1}\right): 3433,3326 v(\mathrm{NH})\left(\mathrm{NH}_{2}\right), 2198 v(\mathrm{CN}), 1615$ $\delta(\mathrm{NH}) .{ }^{1} \mathrm{H}$ NMR $\left(300 \mathrm{MHz}, \mathrm{DMSO}-d_{6}+\mathrm{CDCl}_{3}, \delta, \mathrm{ppm}\right): 4.62(\mathrm{~s}$, br, $\left.2 \mathrm{H}, \mathrm{NH}_{2}\right), 2.53\left(\mathrm{~m}, 4 \mathrm{H}, \mathrm{CH}_{2}\right), 1.76\left(\mathrm{~m}, 4 \mathrm{H}, \mathrm{CH}_{2}\right) .{ }^{13} \mathrm{C}$ NMR $(75$ MHz, DMSO- $\left.d_{6}+\mathrm{CDCl}_{3}, \delta, \mathrm{ppm}\right): 158.1$ (1C, thienyl-C), 133.2 (1C, thienyl-C), $120.4(1 \mathrm{C}$, thienyl-C), $116.1(1 \mathrm{C}, \mathrm{CN}), 113.2(1 \mathrm{C}$, thienyl-C), $24.3\left(1 \mathrm{C},-\mathrm{CH}_{2}^{-}\right), 23.8\left(1 \mathrm{C},-\mathrm{CH}_{2}-\right), 22.7\left(1 \mathrm{C},-\mathrm{CH}_{2}-\right)$, $21.8\left(1 \mathrm{C},-\mathrm{CH}_{2}-\right)$.

2-Amino-4,5-dimethylthiophene-3-carbonitrile (1b): Color: Red-brownish. Yield: 67 \%. M.p.: $140-142{ }^{\circ} \mathrm{C}$. FT-IR $\left(\mathrm{CHCl}_{3}, v\right.$, $\left.\mathrm{cm}^{-1}\right): 3421,3309 v\left(\mathrm{NH}_{2}\right)\left(\mathrm{NH}_{2}\right), 2212 v(\mathrm{CN}), 1632 \delta(\mathrm{NH})$ $\left(\mathrm{NH}_{2}\right) .{ }^{1} \mathrm{H}$ NMR $\left(300 \mathrm{MHz}, \mathrm{DMSO}-d_{6}+\mathrm{CDCl}_{3}, \delta, \mathrm{ppm}\right): 4.62$ (s, br, $\left.2 \mathrm{H}, \mathrm{NH}_{2}\right), 2.16\left(\mathrm{~s}, 3 \mathrm{H}, \mathrm{CH}_{3}\right), 2.07$ (s, 3H, $\left.\mathrm{CH}_{3}\right) .{ }^{13} \mathrm{C}$ NMR $(75 \mathrm{MHz}$, DMSO-d $\left.d_{6}+\mathrm{CDCl}_{3}, \delta, \mathrm{ppm}\right): 159.1(1 \mathrm{C}$, thienyl-C), $129.6(1 \mathrm{C}$, thienyl-C), 120.2 (1C, thienyl-C), 115.9 (1C, CN), 112.5 (1C, thienyl-C), $12.7\left(1 \mathrm{C}, \mathrm{CH}_{3}\right), 12.3\left(1 \mathrm{C}, \mathrm{CH}_{3}\right)$.

2-Amino-4-(4-chlorophenyl)thiophene-3-carbonitrile (1c): Color: Orange. Yield: 88 \%. M.p.: 162-164 ${ }^{\circ} \mathrm{C}$. FT-IR $\left(\mathrm{CHCl}_{3}, \mathrm{v}\right.$, $\left.\mathrm{cm}^{-1}\right): 3421,3309 v(\mathrm{NH})\left(\mathrm{NH}_{2}\right), 2212 v(\mathrm{CN}), 1638 \delta(\mathrm{NH})\left(\mathrm{NH}_{2}\right)$, 816 (C-Cl), 766 (disubstituted phenyl).

European Journal of Chemistry 


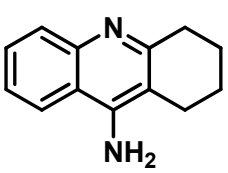

Tacrine

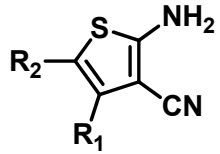

1

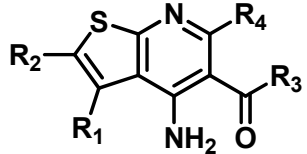

2

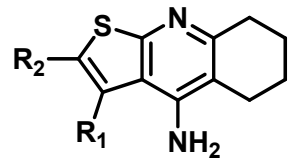

3

Figure 1. Chemical structures of tacrine, starting material $\mathbf{1}$ and the synthesized compounds $\mathbf{2}$ and $\mathbf{3}$.

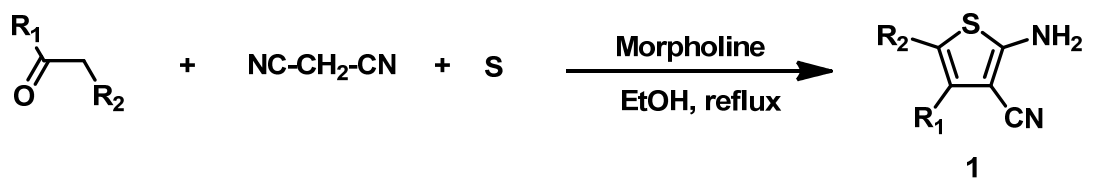

Scheme 1

${ }^{1} \mathrm{H}$ NMR $\left(300 \mathrm{MHz}\right.$, DMSO- $\left.d_{6}+\mathrm{CDCl}_{3}, \delta, \mathrm{ppm}\right): 7.32(\mathrm{~d}, 2 \mathrm{H}, J=$ $8.5 \mathrm{~Hz}, \mathrm{Ar}-\mathrm{H}), 7.04$ (d, 2H, $J=8.5 \mathrm{~Hz}, \mathrm{Ar}-\mathrm{H}), 6.87$ (s, 1H, thienyl$\mathrm{H}), 5.38\left(\mathrm{~s}, \mathrm{br}, 2 \mathrm{H}, \mathrm{NH}_{2}\right) .{ }^{13} \mathrm{C}$ NMR (75 MHz, DMSO-d $d_{6}+\mathrm{CDCl}_{3}, \delta$, ppm): 165.2 (1C, thienyl-C), 137.6 (1C, thienyl-C), 134.7 (1C, Ar-C), 132.1 (1C, Ar-C), 128.1 (2C, Ar-C), 127.8 (2C, Ar-C), 121.2 (1C, thienyl-C), $115.6(1 \mathrm{C}, \mathrm{CN}), 104.0(1 \mathrm{C}$, thienyl-C).

\subsubsection{General method for the Friedländer reaction}

\subsubsection{Preparation of the activated bentonite}

In a reactor equipped with a condenser, a stirrer magnetic and a thermometer was charged a dry bentonite and then concentrated sulfuric acid was added (Mass of $\mathrm{H}_{2} \mathrm{SO}_{4}$ /Mass of Bentonite $=0.3$ ). The mixture was then heated at about $100{ }^{\circ} \mathrm{C}$, the temperature is kept constant during the entire process activation, by means of a water bath. The contact time is determined from the moment when the temperature of the suspension reaches $100{ }^{\circ} \mathrm{C}$. Activated bentonite is then filtered and then washed with distilled water until all traces acid. The wash cycle is completed when the filtrate no longer gives the reaction of sulphates with barium chloride. Bentonite, free of sulphates ions is then dried at 105 to $110^{\circ} \mathrm{C}$, then ground and sieved.

\subsubsection{Procedure for the synthesis of compounds 2 and 3}

To a suspension of catalyst $\left(\mathrm{AlCl}_{3}\right.$ or activated bentonite) in dry 1,2-dichloroethane at room temperature under nitrogen, the corresponding aminothiophene $\mathbf{1}(5 \mathrm{mmol})$ and ketone (10 $\mathrm{mmol})$ were added. The mixture was refluxed until the reaction was complete (monitoring by TLC).

When $\mathrm{AlCl}_{3}$ was used: a solution of $\mathrm{NaOH}(10 \%)$ was added until $\mathrm{pH}=9$ and then, a mixture of water:THF $(v: v, 1: 2)$ was added and the mixture was extracted with dichloromethane. The organic layer was dried, filtered and evaporated. The collected solid was submitted to chromatography $\left(\mathrm{CH}_{2} \mathrm{Cl}_{2}: \mathrm{MeOH}, 9: 1, v: v\right)$ to give the desired compound.

When activated bentonite was used as support catalyst: The mixture was filtered, a solution of water:THF $(1: 2, v: v)$ was added and usually work-up provided the product (Scheme 2 ).

11-Amino-2,3,4,7,8,9-hexahydro[1]benzothieno[2,3-b] quinolin-10(1H)-one (2a): Following the general method for the Friedländer reaction, $\mathrm{AlCl}_{3}(800 \mathrm{mg}$ ) or Activated bentonite $(1000 \mathrm{mg})$ in dry 1,2-dichloroethane $(50 \mathrm{~mL})$ was reacted with cyclohexane-1,3-dione $(10 \mathrm{mmol})$ and aminothiophene 1a (5 mmol). The mixture was refluxed for $24 \mathrm{~h}$. Work-up and chromatography provided compound 2a. Color: Brown. Yield: 67 \%. M.p.: $219-221{ }^{\circ}$ C. FT-IR (KBr, v, cm$\left.{ }^{-1}\right): 3382,3215 v(\mathrm{NH})$ $\left(\mathrm{NH}_{2}\right), 1638 v(\mathrm{CO}), 1589 \delta(\mathrm{NH})\left(\mathrm{NH}_{2}\right), 1328\left(\mathrm{NH}_{2}\right) .{ }^{1} \mathrm{H}$ NMR
(300 MHz, DMSO- $\left.d_{6}, \delta, \mathrm{ppm}\right): 5.08\left(\mathrm{~s}, \mathrm{br}, 1 \mathrm{H}, \mathrm{NH}_{2}\right), 3.1-2.8(\mathrm{~m}$, $\left.4 \mathrm{H}, 2 * \mathrm{CH}_{2}\right), 2.66\left(\mathrm{~m}, 2 \mathrm{H},-\mathrm{CH}_{2}-\mathrm{CO}-\right), 2.53\left(\mathrm{~m}, 2 \mathrm{H},=\mathrm{C}^{-\mathrm{CH}_{2}}\right), 2.12$ (m, $\left.2 \mathrm{H},-\mathrm{CH}_{2}-\mathrm{CH}_{2}-\mathrm{CH}_{2}-\mathrm{CO}-\right), 1.74\left(\mathrm{~m}, 4 \mathrm{H}, 2 * \mathrm{CH}_{2}\right), 1.33$ (s, br, $1 \mathrm{H}$, $\mathrm{NH}_{2}$ linked H-bonding to CO). ${ }^{13} \mathrm{C}$ NMR (75 MHz, DMSO- $d_{6}, \delta$, ppm): 199.7 (1C, CO), 162.9 (1C, -S-C=C(N)-), $161.8(1 \mathrm{C},-\mathrm{N}=\mathrm{C}-$ $\mathrm{CH}_{2}-$ ), 153.1 (1C, =C- $\mathrm{NH}_{2}$ ), 132.7 (1C, $\left.-\mathrm{C}=\mathrm{C}-\mathrm{S}\right), 128.6$ (1C, $-\mathrm{C}=\mathrm{C}-$ $\mathrm{S}), 118.2(1 \mathrm{C}$, thienyl-C=C-C-NH 2$), 112.3(1 \mathrm{C}$, pyridinyl-C-CO), 40.1 (1C, $-\mathrm{CH}_{2}-\mathrm{CO}$ ), 30.8 (1C, $-\mathrm{CH}_{2}-\mathrm{CH}_{2}-\mathrm{CO}$ ), 28.4 (1C, thienyl$\mathrm{CH}_{2}-\mathrm{CH}_{2}-\mathrm{CH}_{2}-\mathrm{CH}_{2}-$ ), 26.9 (1C, thienyl- $\mathrm{CH}_{2}-\mathrm{CH}_{2}-\mathrm{CH}_{2}-\mathrm{CH}_{2}-$ ), 22.7 (1C, thienyl- $\mathrm{CH}_{2}-\mathrm{CH}_{2}-\mathrm{CH}_{2}-\mathrm{CH}_{2}-$ ), 21.4 (1C, thienyl- $\mathrm{CH}_{2}-\mathrm{CH}_{2}-\mathrm{CH}_{2}-$ $\left.\mathrm{CH}_{2}-\right), 20.9$ (1C, pyridinyl- $\left.\mathrm{CH}_{2}-\mathrm{CH}_{2-}\right)$. Anal. calcd. for $\mathrm{C}_{15} \mathrm{H}_{16} \mathrm{~N}_{2} \mathrm{OS}: \mathrm{C}, 66.15 ; \mathrm{H}, 5.92 ; \mathrm{N}, 10.29$. Found: C, 66.08; H, 5.85; N, $10.34 \%$.

4-Amino-2,3-dimethyl-7,8-dihydro-6H-thieno[2,3-b]quinolin5-one (2b): Following the general method for the Friedländer reaction, $\mathrm{AlCl}_{3}(800 \mathrm{mg}$ ) or Activated bentonite $(1000 \mathrm{mg})$ in dry 1,2-dichloroethane $(50 \mathrm{~mL})$ was reacted with cyclohexane1,3-dione $(10 \mathrm{mmol})$ and aminothiophene $\mathbf{1 b}(5 \mathrm{mmol})$. The mixture was refluxed for $24 \mathrm{~h}$. Work-up and chromatography provided compound 2b. Color: Brown. Yield: 71 \%. M.p.: 202$204{ }^{\circ} \mathrm{C}$. FT-IR (KBr, v, cm-1): 3397, $3216 v(\mathrm{NH})\left(\mathrm{NH}_{2}\right), 1638$ $v(\mathrm{CO}), 1587 \delta(\mathrm{NH})\left(\mathrm{NH}_{2}\right), 1422 \delta(\mathrm{CH})\left(\mathrm{CH}_{2}\right) .{ }^{1} \mathrm{H}$ NMR $(300 \mathrm{MHz}$, DMSO- $\left.d_{6}, \delta, \mathrm{ppm}\right): 5.33\left(\mathrm{~s}, \mathrm{br}, 1 \mathrm{H}, \mathrm{NH}_{2}\right), 2.97\left(\mathrm{~m}, 2 \mathrm{H},-\mathrm{CH}_{2}-\mathrm{CO}-\right)$, $2.72\left(\mathrm{~m}, 2 \mathrm{H},=\mathrm{C}-\mathrm{CH}_{2}\right), 2.53\left(\mathrm{~m}, 3 \mathrm{H}, \mathrm{CH}_{3}\right), 2.37\left(\mathrm{~m}, 3 \mathrm{H}, \mathrm{CH}_{3}\right), 2.11$ (m, $2 \mathrm{H},-\mathrm{CH}_{2}-\mathrm{CH}_{2}-\mathrm{CH}_{2}-\mathrm{CO}-$ ), 1.63 (s, br, $1 \mathrm{H}, \mathrm{NH}_{2}$ linked $\mathrm{H}-$ bonding to $\mathrm{CO}$ ). ${ }^{13} \mathrm{C}$ NMR (75 MHz, DMSO- $d_{6}, \delta, \mathrm{ppm}$ ): $201.2(1 \mathrm{C}$, CO), $162.1(1 \mathrm{C},-\mathrm{S}-\mathrm{C}=\mathrm{C}(\mathrm{N})-), 161.6\left(1 \mathrm{C},-\mathrm{N}=\mathrm{C}-\mathrm{CH}_{2}-\right), 152.6(1 \mathrm{C}$, $\left.=\mathrm{C}-\mathrm{NH}_{2}\right), 138.2(1 \mathrm{C},-\mathrm{C}=\mathrm{C}-\mathrm{S}), 125.6(1 \mathrm{C},-\mathrm{C}=\mathrm{C}-\mathrm{S}), 121.9$ (1C, thienyl-C=C-C- $\left.\mathrm{NH}_{2}\right), 112.5\left(1 \mathrm{C}\right.$, pyridinyl-C-CO), $39.9\left(1 \mathrm{C},-\mathrm{CH}_{2}-\right.$ CO), 29.8 (1C, $\left.-\mathrm{CH}_{2}-\mathrm{CH}_{2}-\mathrm{CO}\right), 21.4$ (1C, pyridinyl- $\mathrm{CH}_{2}-\mathrm{CH}_{2}-$ ), 14.8 (1C, $\left.\mathrm{CH}_{3}\right), 13.1\left(1 \mathrm{C}, \mathrm{CH}_{3}\right)$. Anal. calcd. for $\mathrm{C}_{13} \mathrm{H}_{14} \mathrm{~N}_{2} \mathrm{OS}$ : C, 63.39; H, 5.73; N, 11.37. Found: C, 63.43; H, 5.68; N, 10.41\%.

4-Amino-3-(4-chlorophenyl)-7,8-dihydro-6H-thieno[2,3-b] quinolin-5-one (2c): Following the general method for the Friedländer reaction, $\mathrm{AlCl}_{3}(800 \mathrm{mg})$ or Activated bentonite $(1000 \mathrm{mg})$ in dry 1,2-dichloroethane $(50 \mathrm{~mL})$ was reacted with cyclohexane-1,3-dione $(10 \mathrm{mmol})$ and aminothiophene 1c (5 mmol). The mixture was refluxed for $24 \mathrm{~h}$. Work-up and chromatography provided compound 2c. Color: Brown. Yield: 64 \%. M.p.: $234-236^{\circ} \mathrm{C}$. FT-IR (KBr, v, $\left.\mathrm{cm}^{-1}\right)$ : 3396, $3212 v(\mathrm{NH})$ $\left(\mathrm{NH}_{2}\right), 1636 v(\mathrm{CO}), 1587 \quad \delta(\mathrm{NH}) \quad\left(\mathrm{NH}_{2}\right), 831 \quad(\mathrm{C}-\mathrm{Cl}), 765$ (disubstituted phenyl). ${ }^{1} \mathrm{H}$ NMR $\left(300 \mathrm{MHz}, \mathrm{DMSO}-d_{6}, \delta, \mathrm{ppm}\right)$ : $7.36(\mathrm{~m}, 4 \mathrm{H}, \mathrm{Ar}-\mathrm{H}), 6.86\left(\mathrm{~s}, 1 \mathrm{H}\right.$, thienyl-H), $5.28\left(\mathrm{~s}, \mathrm{br}, 1 \mathrm{H}, \mathrm{NH}_{2}\right)$, $2.91\left(\mathrm{~m}, 2 \mathrm{H}, \mathrm{N}=\mathrm{C}-\mathrm{CH}_{2}\right), 2.63\left(\mathrm{~m}, 2 \mathrm{H},-\mathrm{CH}_{2}-\mathrm{CO}-\right), 2.07(\mathrm{~m}, 2 \mathrm{H},-$ $\mathrm{CH}_{2}-\mathrm{CH}_{2}-\mathrm{CH}_{2}-\mathrm{CO}-$ ), 1.42 (s, br, $1 \mathrm{H}, \mathrm{NH}_{2}$ linked H-bonding to $\mathrm{CO}$ ). ${ }^{13} \mathrm{C}$ NMR (75 MHz, DMSO- $\left.d_{6}, \delta, \mathrm{ppm}\right): 198.3$ (1C, CO), 161.8 (1C, $-\mathrm{S}-\mathrm{C}=\mathrm{C}(\mathrm{N})-), 160.1\left(1 \mathrm{C},-\mathrm{N}=\mathrm{C}-\mathrm{CH}_{2}-\right), 154.3\left(1 \mathrm{C},=\mathrm{C}-\mathrm{NH}_{2}\right), 145.9$ (1C, -C=C-S), 136.2 (1C, C-Ar), 135.6 (1C, Cl-C-Ar), 134.2 (2C, 

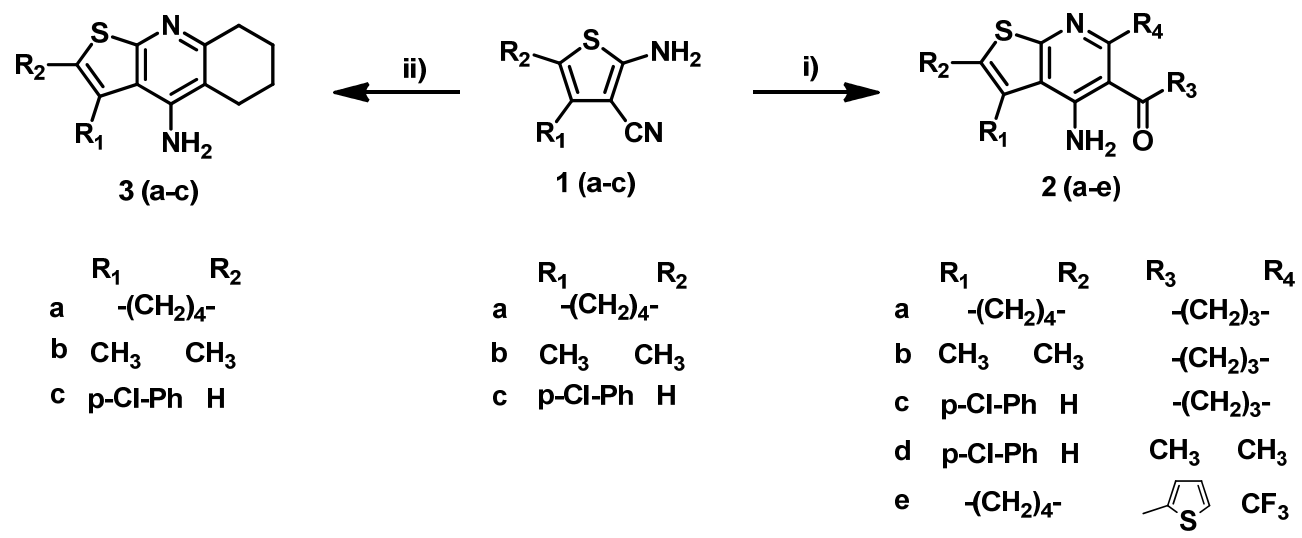

\section{Reagents and conditions i) $\beta$-diketone, catalyst, 1,2-dichloroethane, reflux. ii) cyclohexanone, catalyst, 1,2-dichloroethane, reflux}

Scheme 2

CH-Ar), 129.3 (2C, CH-Ar), 128.7 (1C, -C=C-S), 121.2 (1C, thienyl-C=C-C- $\mathrm{NH}_{2}$ ), 115.2 (1C, pyridinyl-C-CO), $33.8\left(1 \mathrm{C},-\mathrm{CH}_{2}-\right.$ $\mathrm{CO}$ ), 25.4 (1C, - $\mathrm{CH}_{2}-\mathrm{CH}_{2}-\mathrm{CO}$ ), 22.7 (1C, pyridinyl- $\mathrm{CH}_{2}-\mathrm{CH}_{2}-$ ). Anal. Calcd. For $\mathrm{C}_{17} \mathrm{H}_{13} \mathrm{ClN}_{2} \mathrm{OS}$ : C, $62.10 ; \mathrm{H}, 3.98 ; \mathrm{N}, 8.52$. Found: C, $62.14 ; \mathrm{H}, 4.01 ; \mathrm{N}, 8.47 \%$.

1-[4-Amino-3-(4-chlorophenyl)-6-methylthieno [2,3-b] pyridin-5-yl]ethanone (2d): Following the general method for the Friedländer reaction, $\mathrm{AlCl}_{3}(800 \mathrm{mg}$ ) or Activated bentonite $(1000 \mathrm{mg})$ in dry 1,2-dichloroethane $(50 \mathrm{~mL})$ was reacted with cyclohexane-1,3-dione $(10 \mathrm{mmol})$ and aminothiophene 1c (5 mmol). The mixture was refluxed for $24 \mathrm{~h}$. Work-up and chromatography provided compound 2d. Color: Brown. Yield: 73 \%. M.p.: 208-210 ${ }^{\circ}$ C. FT-IR (KBr, v, cm-1): 3349, 3224 v(NH) $\left(\mathrm{NH}_{2}\right), 1635 v(\mathrm{CO}), 1589 \delta(\mathrm{NH}) \quad\left(\mathrm{NH}_{2}\right), 831 \quad(\mathrm{C}-\mathrm{Cl}), 766$ (disubstituted phenyl). ${ }^{1} \mathrm{H}$ NMR (300 MHz, DMSO- $\left.d_{6}, \delta, \mathrm{ppm}\right)$ : $7.41(\mathrm{~m}, 4 \mathrm{H}, \mathrm{ArH}), 6.89$ (s, $1 \mathrm{H}$, thienyl-H), 5.17 (s, br, $\left.1 \mathrm{H}, \mathrm{NH}_{2}\right)$, $2.66\left(\mathrm{~m}, 3 \mathrm{H}, \mathrm{N}=\mathrm{C}-\mathrm{CH}_{3}\right), 2.52\left(\mathrm{~m}, 3 \mathrm{H}, \mathrm{CH}_{3}-\mathrm{CO}-\right), 1.68(\mathrm{~s}, \mathrm{br}, 1 \mathrm{H}$, $\mathrm{NH}_{2}$ linked $\mathrm{H}$-bonding to CO). ${ }^{13} \mathrm{C}$ NMR $\left(75 \mathrm{MHz}, \mathrm{DMSO}-d_{6}, \delta\right.$, ppm): 197.4 (1C, CO), $161.3(1 \mathrm{C},-\mathrm{S}-\mathrm{C}=\mathrm{C}(\mathrm{N})-), 158.6(1 \mathrm{C},-\mathrm{N}=\mathrm{C}-$ $\left.\mathrm{CH}_{3}\right), 154.7\left(1 \mathrm{C},=\mathrm{C}-\mathrm{NH}_{2}\right), 145.9(1 \mathrm{C},-\mathrm{C}=\mathrm{C}-\mathrm{S}), 135.9$ (1C, C-Ar), 135.2 (1C, Cl-C-Ar), 134.2 (2C, CH-Ar), 128.7 (2C, CH-Ar), 127.2 $(1 \mathrm{C},-\mathrm{C}=\mathrm{C}-\mathrm{S}), 121.4 \quad(1 \mathrm{C}$, thienyl-C=C-C-NH$), 115.5 \quad(1 \mathrm{C}$, pyridinyl-C-CO), $17.1\left(1 \mathrm{C}, \mathrm{CH}_{3}\right), 14.2\left(1 \mathrm{C}, \mathrm{CH}_{3}\right)$. Anal. Calcd. For $\mathrm{C}_{16} \mathrm{H}_{13} \mathrm{ClN}_{2} \mathrm{OS}$ : C, 60.66; H, 4.14; N, 8.85 . Found: C, 60.61; H, $4.17 ; \mathrm{N}, 8.91 \%$.

(4-Amino-2-trifluoromethyl-5,6,7,8-tetrahydrobenzo[4,5] thieno[2,3-b]pyridin-3-yl)thiophen-2-yl-methanone (2e): Following the general method for the Friedländer reaction, $\mathrm{AlCl}_{3}(800 \mathrm{mg})$ or Activated bentonite $(1000 \mathrm{mg})$ in dry 1,2dichloroethane $(50 \mathrm{~mL})$ was reacted with 1-thiophen-2ylbutane-1,3-dione $(10 \mathrm{mmol})$ and aminothiophene 1a (5 mmol). The mixture was refluxed for $24 \mathrm{~h}$. Work-up and chromatography provided compound 2e. Color: Yelllow-Brown. Yield: 68 \%. M.p.: $183-185^{\circ} \mathrm{C}$. FT-IR (KBr, v, cm$\left.{ }^{-1}\right): 3376,3253$ $v(\mathrm{NH})\left(\mathrm{NH}_{2}\right), 1672 v(\mathrm{CO}), 1583 \delta\left(\mathrm{NH}_{2}\right)\left(\mathrm{NH}_{2}\right) .{ }^{1} \mathrm{H}$ NMR $(300$ MHz, DMSO- $\left.d_{6}+\mathrm{CDCl}_{3}, \delta, \mathrm{ppm}\right): 7.2(\mathrm{~d}, J=5.2 \mathrm{~Hz}, 1 \mathrm{H},-$ thiophene-H), 6.8-6.5 (m, 2H, -thienyl-H), $5.82\left(\mathrm{~s}, \mathrm{br}, 1 \mathrm{H}, \mathrm{NH}_{2}\right)$, $2.83\left(\mathrm{~m}, 2 \mathrm{H},-\mathrm{CH}_{2}\right), 2.58\left(\mathrm{~m}, 2 \mathrm{H},-\mathrm{CH}_{2}\right), 2.08\left(\mathrm{~m}, 4 \mathrm{H}, 2^{*}-\mathrm{CH}_{2}\right)$, 1.72 (s, br, $1 \mathrm{H}, \mathrm{NH}_{2}$ linked H-bonding to $\mathrm{CO}$ ). ${ }^{13} \mathrm{C}$ NMR (75 MHz, DMSO- $\left.d_{6}, \delta, p p m\right): 196.5(1 \mathrm{C}, \mathrm{CO}), 163.7(1 \mathrm{C},-\mathrm{S}-\mathrm{C}=\mathrm{C}(\mathrm{N})-), 159.2$ $\left(1 \mathrm{C},-\mathrm{N}=\mathrm{C}-\mathrm{CF}_{3}\right), 151.7$ (1C, =C-NH ), $146.8(1 \mathrm{C},-\mathrm{C}=\mathrm{C}-\mathrm{S}), 136.2$, (1C, thienyl-C), 131.2 (1C, thienyl-C), 130.1 (1C, thienyl-C), 128.5 (1C, thienyl-C), 126.3 (1C, $-\mathrm{C}=\mathrm{C}-\mathrm{S}$ ), 125.7 (1C, thienyl$\left.\mathrm{C}=\mathrm{C}-\mathrm{C}-\mathrm{NH}_{2}\right), 118.2\left(1 \mathrm{C}\right.$, pyridinyl-C-CO), $114.2\left(1 \mathrm{C}, \mathrm{CF}_{3}\right), 28.3$ (1C, $-\mathrm{CH}_{2}-\mathrm{CH}_{2}-\mathrm{CH}_{2}-\mathrm{CH}_{2}$ ), 28.1 (1C, $\left.-\mathrm{CH}_{2}-\mathrm{CH}_{2}-\mathrm{CH}_{2}-\mathrm{CH}_{2}-\right), 25.3$ (1C, $-\mathrm{CH}_{2}-\mathrm{CH}_{2}-\mathrm{CH}_{2}-\mathrm{CH}_{2}-$ ), 24.8 (1C, $-\mathrm{CH}_{2}-\mathrm{CH}_{2}-\mathrm{CH}_{2}-\mathrm{CH}_{2}-$ ). Anal. calcd. for $\mathrm{C}_{17} \mathrm{H}_{13} \mathrm{~F}_{3} \mathrm{~N}_{2} \mathrm{OS}$ : C, 58.28; H, 3.74; N, 8.00. Found: C, 58.19; H, 3.82; N, 8.07\%.

11-Amino-1,2,3,4,7,8,9,10-octahydro[1]benzothieno[2,3-b] quinoline (3a): Following the general method for the Friedländer reaction, $\mathrm{AlCl}_{3}$ ( $800 \mathrm{mg}$ ) or Activated bentonite $(1000 \mathrm{mg})$ in dry 1,2-dichloroethane $(50 \mathrm{~mL})$ was reacted with cyclohexanone $(10 \mathrm{mmol})$ and aminothiophene $1 \mathrm{a}(5 \mathrm{mmol})$. The mixture was refluxed for $24 \mathrm{~h}$. Work-up and chromatography provided compound 3a. Color: Brown. Yield: 93 \%. M.p.: $215-217^{\circ} \mathrm{C}$. FT-IR (KBr, v, cm ${ }^{-1}$ ): 3471, 3356 v(NH) $\left(\mathrm{NH}_{2}\right), 1602 \delta(\mathrm{NH})\left(\mathrm{NH}_{2}\right), 1431\left(\mathrm{CH}_{2}\right) .{ }^{1} \mathrm{H}$ NMR $(300 \mathrm{MHz}$, DMSO- $\left.d_{6}, \delta, \mathrm{ppm}\right): 4.62\left(\mathrm{~s}, \mathrm{br}, 2 \mathrm{H}, \mathrm{NH}_{2}\right), 2.93\left(\mathrm{~m}, 2 \mathrm{H}, \mathrm{CH}_{2}\right), 2.81$ $\left(\mathrm{m}, 2 \mathrm{H}, \mathrm{CH}_{2}\right), 2.51\left(\mathrm{~m}, 4 \mathrm{H}, 2 * \mathrm{CH}_{2}\right), 1.96-1.91\left(\mathrm{~m}, 8 \mathrm{H}, 4 * \mathrm{CH}_{2}\right) .{ }^{13} \mathrm{C}$ NMR (75 MHz, DMSO- $\left.d_{6}, \delta, \mathrm{ppm}\right): 159.1(1 \mathrm{C},-\mathrm{S}-\mathrm{C}=\mathrm{C}(\mathrm{N})-), 153.9$ $\left(1 \mathrm{C},-\mathrm{N}=\mathrm{C}-\mathrm{CH}_{2}-\right), 147.7\left(1 \mathrm{C},=\mathrm{C}-\mathrm{NH}_{2}\right), 132.8(1 \mathrm{C},-\mathrm{C}=\mathrm{C}-\mathrm{S}), 131.6$ $(1 \mathrm{C},-\mathrm{C}=\mathrm{C}-\mathrm{S}), \quad 126.1 \quad(1 \mathrm{C}$, thienyl-C=C-C-NH$), 117.3 \quad(1 \mathrm{C}$, pyridinyl-C- $\left.-\mathrm{CH}_{2}\right), 43.7$ (1C, thienyl- $\mathrm{CH}_{2}-\mathrm{CH}_{2}-\mathrm{CH}_{2}-\mathrm{CH}_{2}-$ ), 42.6 (1C, thienyl- $\mathrm{CH}_{2}-\mathrm{CH}_{2}-\mathrm{CH}_{2}-\mathrm{CH}_{2}$ ), 33.3 (1C, pyridinyl- $\mathrm{CH}_{2}-\mathrm{CH}_{2}-$ $\mathrm{CH}_{2}-\mathrm{CH}_{2}-$ ), 31.0 (1C, pyridinyl- $\mathrm{CH}_{2}-\mathrm{CH}_{2}-\mathrm{CH}_{2}-\mathrm{CH}_{2}-$ ), 25.7 (1C, pyridinyl- $\mathrm{CH}_{2}-\mathrm{CH}_{2}-\mathrm{CH}_{2}-\mathrm{CH}_{2}$-), 23.4 (1C, thienyl- $-\mathrm{CH}_{2}-\mathrm{CH}_{2}-\mathrm{CH}_{2}-$ $\mathrm{CH}_{2}$-), 22.6 (1C, thienyl- $\mathrm{CH}_{2}-\mathrm{CH}_{2}-\mathrm{CH}_{2}-\mathrm{CH}_{2}$ ), 21.9 (1C, pyridinyl$\mathrm{CH}_{2}-\mathrm{CH}_{2}-\mathrm{CH}_{2}-\mathrm{CH}_{2}$-). Anal. calcd. for $\mathrm{C}_{15} \mathrm{H}_{18} \mathrm{~N}_{2} \mathrm{~S}: \mathrm{C}, 69.71 ; \mathrm{H}, 7.02$; N, 10.83. Found: C, 69.52; H, 7.08; N, 10.91\%.

2,3-Dimethyl-5,6,7,8-tetrahydrothieno [2,3-b]quinolin-4amine (3b): Following the general method for the Friedländer reaction, $\mathrm{AlCl}_{3}(800 \mathrm{mg}$ ) or Activated bentonite $(1000 \mathrm{mg})$ in dry 1,2-dichloroethane $(50 \mathrm{~mL})$ was reacted with cyclohexanone $(10 \mathrm{mmol})$ and aminothiophene $\mathbf{1 b}(5 \mathrm{mmol})$. The mixture was refluxed for $24 \mathrm{~h}$. Work-up and chromatography provided compound $\mathbf{3 b}$. Color: Grey. Yield: 88 \%. M.p.: $166-168{ }^{\circ} \mathrm{C}$. FT-IR (KBr, $\left.v, \mathrm{~cm}^{-1}\right): 3496,3343 v(\mathrm{NH})$ $\left(\mathrm{NH}_{2}\right), 2922,2857 v(\mathrm{CH})\left(\mathrm{CH}_{3}\right), 1632 \delta(\mathrm{NH})\left(\mathrm{NH}_{2}\right), 1279\left(\mathrm{NH}_{2}\right)$. ${ }_{1}^{1} \mathrm{H}$ NMR $\left(300 \mathrm{MHz}, \mathrm{DMSO}-d_{6}+\mathrm{CDCl}_{3}, \delta, \mathrm{ppm}\right): 4.58(\mathrm{~s}, \mathrm{br}, 2 \mathrm{H}$, $\left.\mathrm{NH}_{2}\right), 2.91\left(\mathrm{~m}, 2 \mathrm{H}, \mathrm{CH}_{2}\right), 2.53\left(\mathrm{~s}, 3 \mathrm{H}, \mathrm{CH}_{3}\right), 2.41\left(\mathrm{~s}, 3 \mathrm{H}, \mathrm{CH}_{3}\right)$, 1.96-1.91 (m, 6H, $\left.3 * \mathrm{CH}_{2}\right) .{ }^{13} \mathrm{C}$ NMR (75 MHz, DMSO- $\left.d_{6}, \delta, \mathrm{ppm}\right):$ $158.3(1 \mathrm{C},-\mathrm{S}-\mathrm{C}=\mathrm{C}(\mathrm{N})-), 153.8$ (1C, $\left.-\mathrm{N}=\mathrm{C}-\mathrm{CH}_{2}-\right), 147.2$ (1C, =C$\mathrm{NH}_{2}$ ), 133.1 (1C, $\left.-\mathrm{C}=\mathrm{C}-\mathrm{S}\right), 123.6$ (1C, $\left.-\mathrm{C}=\mathrm{C}-\mathrm{S}\right), 126.3$ (1C, thienyl$\left.\mathrm{C}=\mathrm{C}-\mathrm{C}-\mathrm{NH}_{2}\right), 113.5\left(1 \mathrm{C}\right.$, pyridinyl- $\left.\mathrm{C}-\mathrm{CH}_{2}\right), 34.3\left(1 \mathrm{C},-\mathrm{CH}_{2}-\mathrm{CH}_{2}-\right.$ $\left.\mathrm{CH}_{2}-\mathrm{CH}_{2}-\right)$, 33.1 (1C, $\left.-\mathrm{CH}_{2}-\mathrm{CH}_{2}-\mathrm{CH}_{2}-\mathrm{CH}_{2}-\right), 27.3\left(1 \mathrm{C},-\mathrm{CH}_{2}-\mathrm{CH}_{2}-\right.$ $\mathrm{CH}_{2}-\mathrm{CH}_{2}-$ ), 23.5 (1C, $-\mathrm{CH}_{2}-\mathrm{CH}_{2}-\mathrm{CH}_{2}-\mathrm{CH}_{2}-$ ), $14.8\left(1 \mathrm{C},-\mathrm{CH}_{3}\right), 14.1$ (1C, $-\mathrm{CH}_{3}$ ). Anal. calcd. for $\mathrm{C}_{13} \mathrm{H}_{16} \mathrm{~N}_{2} \mathrm{~S}: \mathrm{C}, 67.20 ; \mathrm{H}, 6.93 ; \mathrm{N}, 12.07$. Found: C, 67.13; H, 7.04; N, 12.26\%.

4-Amino-3-(4-chlorophenyl)-5,6,7,8-tetrahydrothieno[2,3b]quinoline (3c): Following the general method for the Friedländer reaction, $\mathrm{AlCl}_{3}(800 \mathrm{mg}$ ) or Activated bentonite $(1000 \mathrm{mg})$ in dry 1,2-dichloroethane $(50 \mathrm{~mL})$ was reacted with cyclohexanone $(10 \mathrm{mmol})$ and aminothiophene $1 \mathrm{c}(5 \mathrm{mmol})$. 
Table 1. Percentage conversion of compound 2 and $\mathbf{3}$ in the presence of catalytic amount of $\mathrm{AlCl}_{3}$ and acid activated bentonite

\begin{tabular}{|c|c|c|c|c|c|c|c|c|c|}
\hline Compound & & $2 a$ & $2 b$ & $2 \mathrm{c}$ & $2 d$ & $2 \mathrm{e}$ & $3 a$ & $3 b$ & $3 c$ \\
\hline Yield (\%) & $\mathrm{AlCl}_{3}$ used as a support catalyst & 52 & 58 & 55 & 62 & 53 & 78 & 83 & 77 \\
\hline & Activated bentonite used as a support catalyst & 67 & 71 & 64 & 73 & 68 & 93 & 88 & 86 \\
\hline
\end{tabular}
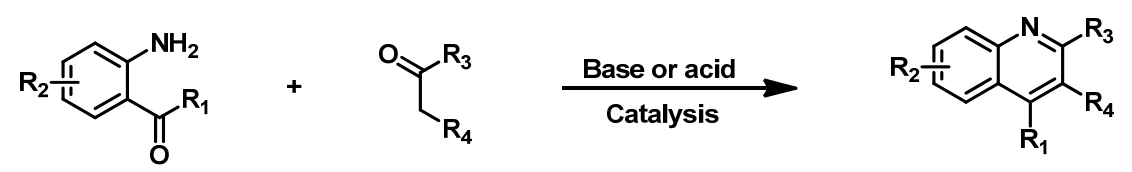

Scheme 3

The mixture was refluxed for $24 \mathrm{~h}$. Work-up and chromatography provided compound 3c. Color: Orange. Yield: 86 \%. M.p.: $177-179{ }^{\circ} \mathrm{C}$. FT-IR (KBr, v, cm$\left.{ }^{-1}\right): 3481,3346 v(\mathrm{NH})$ $\left(\mathrm{NH}_{2}\right), 1621 \delta(\mathrm{NH})\left(\mathrm{NH}_{2}\right), 1447\left(\mathrm{CH}_{2}\right), 831(\mathrm{C}-\mathrm{Cl}) .{ }^{1} \mathrm{H}$ NMR $(300$ MHz, DMSO- $\left.d_{6}, \delta, \mathrm{ppm}\right): 7.37(\mathrm{~m}, 4 \mathrm{H}, \mathrm{Ar}-\mathrm{H}), 7.01(\mathrm{~s}, 1 \mathrm{H}$, thienyl$\mathrm{H}), 4.54\left(\mathrm{~s}, \mathrm{br}, 2 \mathrm{H}, \mathrm{NH}_{2}\right), 2.97\left(\mathrm{~m}, 2 \mathrm{H},-\mathrm{N}=\mathrm{C}-\mathrm{CH}_{2}\right), 2.51(\mathrm{~m}, 2 \mathrm{H}$, $\left.=\mathrm{C}-\mathrm{CH}_{2}\right), 1.93\left(\mathrm{~m}, 4 \mathrm{H}, 2 * \mathrm{CH}_{2}\right) .{ }^{13} \mathrm{C}$ NMR $\left(75 \mathrm{MHz}, \mathrm{DMSO}-d_{6}, \delta\right.$, ppm): $159.1(1 \mathrm{C},-\mathrm{S}-\mathrm{C}=\mathrm{C}(\mathrm{N})-), 155.9\left(1 \mathrm{C},-\mathrm{N}=\mathrm{C}-\mathrm{CH}_{2}-\right), 147.2(1 \mathrm{C}$, $\left.=\mathrm{C}-\mathrm{NH}_{2}\right), 135.8(1 \mathrm{C},-\mathrm{C}=\mathrm{C}-\mathrm{S}), 134.3(1 \mathrm{C}, \mathrm{C}-\mathrm{Ar}), 133.7$ (1C, Cl-C$\mathrm{Ar}$ ), 131.2 (2C, CH-Ar), 129.1 (2C, CH-Ar), 120.2 (1C, -C=C-S), 115.9 (1C, thienyl-C=C-C-NH 2$), 113.8$ (1C, pyridinyl- $\mathrm{C}-\mathrm{CH}_{2}$ ), $33.7\left(1 \mathrm{C},-\mathrm{CH}_{2}-\mathrm{CH}_{2}-\mathrm{CH}_{2}-\mathrm{CH}_{2}-\right), 23.2$ (1C, $-\mathrm{CH}_{2}-\mathrm{CH}_{2}-\mathrm{CH}_{2}-\mathrm{CH}_{2}-$ ), $22.7\left(1 \mathrm{C},-\mathrm{CH}_{2}-\mathrm{CH}_{2}-\mathrm{CH}_{2}-\mathrm{CH}_{2}-\right), 22.4\left(1 \mathrm{C},-\mathrm{CH}_{2}-\mathrm{CH}_{2}-\mathrm{CH}_{2}-\mathrm{CH}_{2}-\right)$. Anal. calcd. for $\mathrm{C}_{17} \mathrm{H}_{15} \mathrm{ClN}_{2} \mathrm{~S}$ : C, 64.84; $\mathrm{H}, 4.80 ; \mathrm{N}, 8.90$. Found: C, 64.79; H, 4.75; N, 8.97\%.

\section{Results and discussion}

Different synthetic approaches for the preparation of aminopyridine rings have been reported, the Friedländer reaction being one of the simplest and most efficient methods [11]. Friedländer reaction is a base or acid catalysed condensation of an aromatic 2-amino substituted carbonyl compounds with carbonyl derivatives containing a reactive $\alpha$ methylene group (Scheme 3).

Many studies have been undertaken with the aim of developing new catalysts operating under milder conditions. The usual acid catalyst promoted Friedländer reaction was Lewis acid such as $\mathrm{Al}_{2} \mathrm{O}_{3}, \mathrm{SiO}_{2}$ and $\mathrm{AlCl}_{3}$.

Clays such as acid activated bentonites have been used as solid catalyst supports for a number of organic synthesis [1215]. The advantages of using these catalysts reside in the association of Lewis and Bronsted site acids, the good absorptive capacity and the simple work-up procedure as the separation of the spent catalyst is achieved by filtration. Otherwise, they have considerable potential as environmental friendly solid acid catalysts and they can be regenerated and reused.

In this present paper, we report our recent studies on the synthesis of 4-aminothienoquinolinones 2 and their tacrine analogues derivatives $\mathbf{3}$ and we are interested in the development of environmentally friendly catalyst such as activated bentonite in the Friedländer reaction. The results of the Friedländer reaction of substituted 2-aminothiophene-3carbonitrile with some carbonyl derivatives containing a reactive $\alpha$-methylene group were depicted outline.

The starting material 2-aminothiophene-3-carbonitrile $\mathbf{1}$ was prepared in one step from corresponding ketones by using versatile Gewald reaction [16,17] (Scheme 1).

The synthesis of the 4-aminothienoquinolinones 2 and thienotacrine $\mathbf{3}$ were performed by using Friedländer reaction. Precursor 1 reacted with $\beta$-dicarbonyl compounds or cyclohexanone under conditions i) or ii) to give respectively products $\mathbf{2}$ and $\mathbf{3}$ (Scheme 2).

The remarkable utility of clays in various reported organic transformations, motivated us to initially test the use of acid activated bentonite as catalyst in this field of reaction. In fact, we found that the use of $1000 \mathrm{mg}$ activated bentonite for 10 mmol of cyclohexanone (or diketone) under dichloroethane reflux for 24 hours afforded thienotacrine $\mathbf{3}$ (or thienoquinolinone 2) in high to good yield as depicted in Table 1. However, attempts to use a superstoichiometric amount of Aluminium chloride ( 4 or 5 equiv.) led to lower yield. The excellent result achieved using acid activated bentonite could be probably explained by high absorptive capacity and the efficient coordination of catalyst and reactants.

\section{Conclusion}

In order to investigate the biological effects of structural modification of tacrine, a simple and green one-pot process was developed for the synthesis of 4-aminothienoquinolinones 2 and thienotacrine $\mathbf{3}$ derivatives via Friedländer condensation reaction promoted by an environmentally friendly catalyst such as bentonite.

\section{Acknowledgements}

The authors extend their appreciation to The Deanship of Scientific Research at Qassim University for the financial support of this work (Project No: 1799).

\section{References}

[1]. Summers, W. K.; Majovsky, L. V.; Marsh, G. M.; Tachiki, K.; Kling, A. N. Engl. J. Med. 1986, 315, 1241-1287

[2]. Sahakian, B. J.; Owen, A. M.; Morant, N. J.; Eagger, S. A.; Boddington, S.; Crayton, L.; Crockford, H. A.; Crooks, M.; Hill, K.; Levy, R. Psychopharmacology 1993, 110, 395-401.

[3]. Drukarch, B.; Kits, K. S.; Van der Meer, E. G.; Lodder, J. C.; Stoof, J. C. Eur. J. Pharmacol. 1987, 141, 153-157.

[4]. Augry, F.; Darch, A.; De Routou, J.; Guelfi, M. C.; Forette, F. J. Pharm Clin. 1997, 16, 183-201.

[5]. Dridi, K.; El Efrit, M. L.; Zantour, H. J. Soc. Chim. Tunisie 1999, 5, 387 392.

[6]. Dridi, K.; El Efrit, M. L.; Zantour, H. J. Soc. Chim. Tunisie 2001, 4, 957963.

[7]. Gutschow, M.; Kuerschner, L.; Neumann, U.; Peitsch, M.; Loser, R.; Koglin, N.; Eger, K. J. Med. Chem. 1999, 42, 5437-5447.

[8]. Fugita, M.; Seki, T.; Ikeda, N. Bioorg. Med. Chem. Lett. 2002, 12, 18971900.

[9]. Harza, K.; Saravanan, J.; Mohan, S. Asian J. Chem. 2007, 19, 3541-3544.

[10]. Narlawar, R.; Lane, J. R.; Doddareddy, M.; Lin, J.; Brussee, J.; Ijzerman, A. P. J. Med. Chem. 2010, 53, 3028-3037.

[11]. Marco-Contelles, J.; Perez-Mayoral, E.; Samadi, A.; Carreiras, M. C.; Soriano, E. Chem. Rev. 2009, 109, 2652-2671.

[12]. Laszlo, P.; Mathy, A. Hel. Chim. Acta 1987, 70, 577-586.

[13]. Choudary, B. M.; Kantam, M. L.; Rao, K. K.; Santhi, P. L. Appl. Catal. A. Gen. 1997, 149, 257-264.

[14]. Yoo, J. W.; Lee, C. W.; Jeong, H. C.; Park, Y. K.; Park, S. E. Catal. Today 2000, 60, 255-261.

[15]. Inui, K.; Kurabayashi, T.; Sato, S.; Ichikawa, N. J. Mol. Catal. A 2006 216, 147-155.

[16]. Gewald, K.; Schinke, E.; Bohcher, H. Chem. Ber. 1966, 99, 94-100.

[17]. Eller, G. A.; Wolfgang, H. Molecules 2006, 11, 371-376. 